\title{
LAS BARTRAMIACEAE (MUSCI) DEL VALLE DE MÉXICO, MÉXICO
}

\author{
Ángeles Cárdenas S. \\ Instituto de Biología, UNAM \\ Apartado postal $70-233$ \\ 04510 México, D.F.
}

\begin{abstract}
RESUMEN
Se presenta una lista de los representantes de la familia Bartramiaceae existentes en el Valle de México que incluye 12 especies, o sea, 39\% del total de 31 especies registradas para el país. Se proporcionan datos de las colectas examinadas, así como la información ambiental correspondiente. Son plantas que crecen preferentemente sobre suelo o rocas, en elevaciones medias y altas, en casi todos los tipos de vegetación presentes en el área de estudio.
\end{abstract}

\section{ABSTRACT}

A list of the members of the family Bartramiaceae occuring in the Valley of Mexico is presented. The family is represented in the area by 12 species amounting to 39 per cent of those recorded (31 species) for the country. Habitat data and other pertinent information is provided for all specimens examined. Members of this family grow mostly on soil and rocks, at intermediate or high elevations in almost all types of vegetation known in the area of study.

\section{INTRODUCCIÓN}

En México la familia Bartramiaceae incluye siete géneros con 31 especies que se distribuyen en elevaciones medias y altas, sobre suelo o rocas (Griffin, 1994). Es una familia morfológicamente muy diversa, pero al mismo tiempo de las más distintivas por sus tallos tomentosos, especialmente en la base; la costa es fuerte, percurrente a largamente excurrente; las células son papilosas en uno o en ambos extremos y sus cápsulas son globosas o semiglobosas, con frecuencia surcadas o menos frecuentemente, rugosas. En el Valle de México se encuentran en un perfil altitudinal amplio que va de los 2230 a los $4020 \mathrm{~m}$ y se localizan en casi todos los tipos de vegetación registrados para la zona, en donde crecen principalmente sobre suelo o rocas volcánicas, en sitios perturbados.

El estudio de las Bartramiaceae es parte del proyecto "Los Musgos del Valle de México" que ha sido el punto central de las investigaciones florísticas de la autora durante los últimos años. La región es florísticamente muy diversa y tal vez es la zona mejor colectada del país (Cárdenas, 1988). Los resultados, dados a conocer en los trabajos de Cárdenas (1989, 1995 a, 1995 b, 1997, 1999) y Cárdenas y Delgadillo $(1992,1994)$ enlistan alrededor de 300 taxa de musgos para esta cuenca. 


\section{MATERIAL Y MÉTODO}

Durante esta investigación se recolectaron muestras en el Valle de México y se revisaron los ejemplares de Bartramiaceae de la Colección Briológica del Herbario Nacional (MEXU). En total se estudiaron alrededor de 150 ejemplares provenientes de varios tipos de vegetación que, según la nomenclatura de Rzedowski y Rzedowski (1979), son: bosque de Abies, bosque mesófilo de montaña, bosque de Pinus, bosque de Quercus, bosque de Juniperus, matorral de Quercus, pastizales y matorral xerófilo.

\section{RESULTADOS Y DISCUSIÓN}

En el Valle de México la familia Bartramiaceae está representada por 12 especies (una de ellas con dos subespecies). En el listado que se presenta a continuación los taxa se mencionan por orden alfabético, con el nombre científico seguido del número de colecta de la autora, tipo de vegetación, microhábitat, altitud, distribución local y algunos comentarios sobre su situación taxonómica. El material de otras personas está indicado por el apellido y el número de colecta correspondientes. Los registros en la literatura van acompañados de una referencia.

Anacolia laevisphaera (Tayl.) Flow. 1956, 2660, 2749, 3677; Delgadillo 351, 418; Vivas 236, 322, 346. Bosque de Abies; Pinus; Quercus; pino-encino; Juniperus; matorral de encino; xérofilo; zacatonal de Muhlenbergia; zona alpina. Sobre arena, suelo, humus o rocas, en lugares soleados, expuestos o sombreados y secos, húmedos o mojados. 2230-3700 m s.n.m. Distrito Federal, Estado de México e Hidalgo.

Bartramia brevifolia Brid. 1405b, 4319, 4382, 4409; Delgadillo 413, 415; Castillo 78a. Bosque de Abies; Pinus; de Pinus y Quercus; zona alpina. Sobre suelo o roca, en lugares soleados, expuestos o sombreados y secos o húmedos. 2900-3950 m s.n.m. Distrito Federal, Estado de México e Hidalgo. Crum (1951) registró a B. glaucula Card. y Griffin (1994) citó a B. schimperi C. M. para el Distrito Federal y el Estado de México. Fransén (1995), sin embargo, las considera como sinónimos de B. brevifolia.

Bartramia potosica Mont. 1420, 3640, 4133; Delgadillo 2108, 2114a, 2127a. Bosque de Pinus; Quercus; zona alpina. Sobre arena, suelo o roca, en lugares expuestos o sombreados y secos, húmedos o mojados. 2830-4020 m s.n.m. Distrito Federal, Estado de México e Hidalgo. Los ejemplares citados arriba no tienen estructuras sexuales por lo que no pudieron ubicarse en alguna de las subespecies reconocidas por Fransén (1995). Los especímenes del Valle de México originalmente identificados como B. mathewsii pertenecen a B. potosica; la primera no parece estar presente en nuestra área. B. glauca Lor. y B. ithyphylla Brid. son sinónimos.

Bartramia potosica Mont. ssp. potosica. 4757. Zona alpina. Roca cubierta por suelo, lugares sombreados y mojados. 3420-3460 m s.n.m. Estado de México. 
Bartramia potosica Mont. ssp. synoica Fransén. Delgadillo 2081; Vivas 240. Bosque de Abies; zona alpina. Suelo, en lugares expuestos o mojados. 3000 m s.n.m. Distrito Federal y Estado de México.

Breutelia chrysocoma var. major (Schimp. ex Besch.) Broth. Crum (1951) la incluye para el Distrito Federal como B. arcuata Schimp. var. major Besch. Griffin (1994) la excluye de la flora mexicana provisionalmente, pero las razones para hacerlo no son totalmente claras.

Breutelia inclinata (Hampe \& Lor.) Jaeg. 2742, 4075; Sharp 65. Bosque de Quercus. Sobre suelo o roca, en lugares expuestos y secos. $2410-2830$ m s.n.m. Distrito Federal, Estado de México e Hidalgo.

Breutelia subarcuata (C.M.) Schimp. 1612a, 3486, 3637; Delgadillo 1300, 1309; Alfaro 625. Bosque de Abies; Pinus; Quercus; matorral xerófilo; zona alpina. Sobre suelo o roca, en lugares soleados, expuestos o sombreados y secos, húmedos o mojados. 24103660 m s.n.m. Estado de México e Hidalgo.

Flowersia campylopus (Schimp. ex C. M.) Griffin \& Buck. 1404, 2927; Amable 1491; Delgadillo 402, 5149. Bosque de Abies; Pinus; Quercus; matorral de Quercus. Rocas cubiertas por suelo, en lugares expuestos o sombreados y secos o mojados. 24003700 m s.n.m. Distrito Federal, Estado de México e Hidalgo.

Philonotis cernua (Wils.) Griffin \& Buck. 2560, 2570; Amable1624. Bosque de Pinus. Sobre suelo, lugares expuestos o sombreados y húmedos. Distrito Federal y Estado de México.

Philonotis corticata Crum \& Griffin. 1422, 1987; Delgadillo 1321, 2168, 2169a. Bosque de Pinus; zona alpina. Rocas cubiertas por suelo o arena, lugares expuestos o sombreados y húmedos o mojados. 3800-4020 m s.n.m. Estado de México. Los ejemplares de Philonotis scabrifolia (Hook. f. \& Wils.) Braithw. registrados por Delgadillo (1970) corresponden a $P$. corticata.

Philonotis fontana (Hedw.) Brid. Sosa 108, 109; Vivas 144. Bosque de Abies. Sobre suelo o roca, lugares expuestos o sombreados y secos o mojados. 3100-3400 m s.n.m. Distrito Federal e Hidalgo.

Philonotis hastata (Duby) Wijk \& Marg. 4403. Bosque de Pinus. Sobre rocas en lecho de arroyo, lugares expuestos, sumergidos. Estado de México.

Philonotis uncinata (Schwaegr.) Brid. 4410. Bosque de Pinus. Sobre roca, lugares expuestos, sumergidos. Estado de México.

Bartramia microstoma Mitt. fue citada del Distrito Federal (Griffin, 1994), pero Fransén (1995) la enlistó con las especies neotropicales dudosas o excluidas, aparentemente porque 
no estudió el ejemplar tipo. En cualquier caso, la especie debe considerarse en estudios posteriores para determinar su validez taxonómica y su presencia en México.

Actualmente se reconocen cerca de 300 especies y unidades infraespecíficas de musgos para el Valle de México, por lo que las Bartramiaceae totalizan alrededor de $4 \%$ de esa flora. Sin embargo, como sucede con las Funariales, Pottiaceae o con los musgos pleurocárpicos, el número relativo de taxa de Bartramiaceae es alto, pues representa 39\% de las especies de la misma familia registradas para el país. La abundancia y la frecuencia de sus componentes en el Valle de México parecen ser favorecidas por la presencia de numerosos microambientes que resultan de la continua perturbación en el área. En el Valle de México las Bartramiaceae, al igual que las Funariales y Pottiaceae, son musgos que pueden invadir sitios abiertos por la caída de árboles, la construcción de caminos o por la presencia de campos de cultivo abandonados. Estas plantas pueden encontrarse en espacios sombreados y húmedos, como parte de las comunidades que caracterizan a los primeros estados sucesionales. Es frecuente observar, por ejemplo, colonias de Philonotis spp. en taludes de carreteras o en los márgenes de arroyos, acompañando a fanerógamas herbáceas y otras briofitas propias de esos sitios.

\section{AGRADECIMIENTOS}

Al Dr. Claudio Delgadillo M. por su apoyo y la revisión crítica del manuscrito y a los revisores anónimos por sus valiosos comentarios.

\section{LITERATURA CITADA}

Cárdenas S., A. 1988. Los musgos cleistocárpicos del Valle de México, México. Bryologist 91: 214216.

Cárdenas S., A. 1989. Nuevos registros para la flora de musgos de México y del Valle de México. Anales Inst. Biol. UNAM. Ser. Bot. 58: 93-95.

Cárdenas S., A. 1995 a. El orden Funariales en el Valle de México, México. Evansia 12: 9-12.

Cárdenas S., A. 1995 b. Las Pottiaceae (Musci) del Valle de México, México. Acta Bot. Mex. 33: 5161.

Cárdenas S., A. 1997. Musgos del Valle de México: Pico La Biznaga (Atizapán de Zaragoza). Evansia 14: $53-56$.

Cárdenas S., A. 1999. Los musgos pleurocárpicos del Valle de México, México. Trop. Bryol. 16: 109116.

Cárdenas S., A. y C. Delgadillo M. 1992. Los musgos de la Sierra de Alcaparrosa, México. Acta Bot. Mex. 17: 23-33.

Cárdenas S., A. y C. Delgadillo M. 1994. Lorentziella imbricata and Oreoweisia delgadilloi in Mexico. Bryologist 97: 85-86.

Crum, H. A. 1951. The Appalachian-Ozarkian element in the moss flora of Mexico with a check-list of all know Mexican mosses.Tesis de doctorado, University of Michigan. Ann Arbor. 504 pp.

Delgadillo M., C. 1970. Phytogeographic studies on alpine mosses of Mexico. Bryologist 74: 331-346.

Fransén, S. 1995. A taxonomic revision of neotropical Bartramia section Vaginella. Lindbergia 20: 147179. 
Cárdenas: Las Bartramiaceae del Valle de México

Griffin III, D. 1994. Bartramiaceae. In: Sharp, A. J., H. A. Crum y P. M. Eckel (eds.) The moss flora of Mexico. Mem. New York Bot. Gard. 69: 537-574.

Rzedowski, J. y G.C. de Rzedowski. 1979. Flora fanerogámica del Valle de México. Vol. 1. CECSA. México, D.F. 402 pp.

Recibido en septiembre de 1999.

Aceptado en marzo de 2000. 